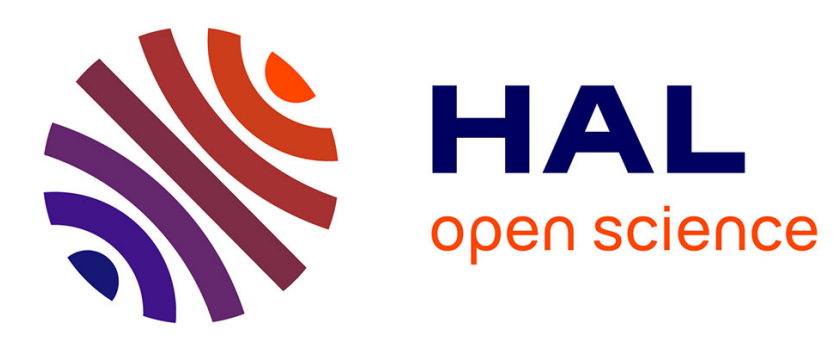

\title{
About Folding-Unfolding Cuts and Cuts Modulo
}

Gilles Dowek

\section{To cite this version:}

Gilles Dowek. About Folding-Unfolding Cuts and Cuts Modulo. RR-4004, INRIA. 2000. inria00072640

\section{HAL Id: inria-00072640 \\ https://hal.inria.fr/inria-00072640}

Submitted on 24 May 2006

HAL is a multi-disciplinary open access archive for the deposit and dissemination of scientific research documents, whether they are published or not. The documents may come from teaching and research institutions in France or abroad, or from public or private research centers.
L'archive ouverte pluridisciplinaire HAL, est destinée au dépôt et à la diffusion de documents scientifiques de niveau recherche, publiés ou non, émanant des établissements d'enseignement et de recherche français ou étrangers, des laboratoires publics ou privés. 
INSTITUT NATIONAL DE RECHERCHE EN INFORMATIQUE ET EN AUTOMATIQUE

\section{About folding-unfolding cuts and cuts modulo}

Gilles Dowek

$N^{\circ} 4004$

Septembre 2000

THÈME 2 



\title{
RINRIA
}

\section{About folding-unfolding cuts and cuts modulo}

\author{
Gilles Dowek* \\ Thème 2 - Génie logiciel \\ et calcul symbolique \\ Projet LogiCal \\ Rapport de recherche $n^{\circ} 4004$ - Septembre 2000 - 10 pages
}

\begin{abstract}
We show in this note that cut elimination in deduction modulo subsumes cut elimination in deduction with the folding and unfolding rules.
\end{abstract}

Key-words: Cut elimination, deduction modulo, folding, unfolding.

* Gilles.Dowek@inria.fr http://logical.inria.fr/ ${ }^{\sim}$ dowek 


\section{Les coupures par pliage-dépliage et les coupures modulo}

Résumé : On montre que l'élimination des coupures en déduction modulo généralise l'élimination des coupures avec des règles de pliage et de dépliage.

Mots-clés : Élimination des coupures, déduction modulo, pliage, dépliage. 
In first-order natural deduction, a cut is a sequence formed with an introduction rule followed by an elimination rule. This notion can be extended to deal with the axioms of some theories, e.g. type theory, set theory, the Stratified Foundations, ... Prawitz [10] proposes a rather uniform way to extend the notion of cut by first extending first-order natural deduction with two rules: folding and unfolding (called $\lambda$-introduction and $\lambda$-elimination by Prawitz) and then considering a sequence of such rules as a new form of cut (see also $[2,9,1,3,8])$. We have recently proposed another way to extend deduction called deduction modulo $[5,7]$ where propositions equivalent modulo a congruence are identified. Identifying propositions this way extends the notion of cut. We show in this note that deduction modulo subsumes deduction with the folding and unfolding rules.

\section{Deduction with the folding and unfolding rules}

In this note, we consider a fixed theory $\mathcal{T}$ formed with axioms

$$
\forall x_{1} \ldots \forall x_{n}\left(P_{i} \Leftrightarrow Q_{i}\right)
$$

where the $P_{i}$ 's are atomic propositions and the $Q_{i}$ 's arbitrary propositions.

We say that a proposition $B$ folds to an atomic proposition $A$ (resp. that $A$ unfolds to $B$ ) if $A=\theta P_{i}$ and $B=\theta Q_{i}$ for some axiom $\forall x_{1} \ldots \forall x_{n}\left(P_{i} \Leftrightarrow Q_{i}\right)$ of $\mathcal{T}$ and some substitution $\theta$. We assume that $\mathcal{T}$ is such that an atomic proposition unfolds to at most one proposition.

The axioms of $\mathcal{T}$ can be replaced by the deduction rules

$$
\begin{aligned}
& \frac{\Gamma \vdash \theta Q_{i}}{\Gamma \vdash \theta P_{i}} \text { folding } \\
& \frac{\Gamma \vdash \theta P_{i}}{\Gamma \vdash \theta Q_{i}} \text { unfolding }
\end{aligned}
$$

and it is easy to check that a sequent $\mathcal{T}, \Gamma \vdash A$ can be proved in first-order natural deduction if and only if the sequent $\Gamma \vdash A$ can be proved in deduction with the folding and unfolding rules. Indeed, any instance of the folding and unfolding rules can be simulated using an axiom of $\mathcal{T}$ and the axioms of $\mathcal{T}$ can be proved in deduction with the folding and unfolding rules.

Since an atomic proposition unfolds to at most one proposition, a sequence formed with a folding and an unfolding rule has the form

$$
\frac{\frac{\ldots}{\Gamma \vdash B}}{\frac{\Gamma \vdash A}{\Gamma \vdash B} \text { unfolding }}
$$

and can be reduced to

$$
\frac{\cdots}{\Gamma \vdash B}
$$


Such a sequence is thus called a folding-unfolding cut.

Cut elimination terminates for some theories $\mathcal{T}$, but it does not for others.

\section{Deduction modulo}

In deduction modulo, a theory is formed with a set of axioms $\Gamma$ and a congruence $\equiv$ on propositions. Here, the congruence is the smallest congruence identifying $P_{i}$ and $Q_{i}$ for each $i$.

The deduction rules take this congruence into account. For instance, the modus ponens is not stated as usual

$$
\frac{\Gamma \vdash A \Rightarrow B \quad \Gamma \vdash A}{\Gamma \vdash B}
$$

as the first premise need not be exactly $A \Rightarrow B$ but may be only congruent to this proposition, hence it is stated

$$
\frac{\Gamma \vdash C \quad \Gamma \vdash A}{\Gamma \vdash B} \text { if } C \equiv A \Rightarrow B
$$

All the rules of intuitionistic natural deduction may be stated in a similar way (figure 1).

A cut in deduction modulo is, like in first-order logic, a sequence formed with a introduction and an elimination rule. Cut elimination terminates for some congruences, but it does not for others.

\section{Proof-terms}

We use a functional notation for proofs. To each proof of a sequent $A_{1}, \ldots, A_{n} \vdash B$ in firstorder natural deduction, we associate a proof-term whose free variables are among $\alpha_{1}, \ldots, \alpha_{n}$. Proofs built with the axiom rules using the axiom $A_{i}$ are written $\alpha_{i}$, proofs built with the introduction and elimination rules of the implication are written $\lambda \alpha \pi$ and $\left(\pi \pi^{\prime}\right)$, proofs built with the introduction and elimination rules of the conjunction are written $\left\langle\pi, \pi^{\prime}\right\rangle, f s t(\pi)$ and $\operatorname{snd}(\pi)$, proofs built with the introduction and elimination rules of the disjunction are written $i(\pi), j(\pi)$ and $\left(\delta \pi_{1} \alpha \pi_{2} \beta \pi_{3}\right)$, proofs built with the elimination rule of the contradiction are written (botelim $\pi$ ) proofs built with the introduction and elimination rules of the universal quantifier are written $\lambda x \pi$ and $(\pi t)$ and proofs built with the introduction and elimination rules of the existential quantifier are written $\langle t, \pi\rangle$ and (exelim $\left.\pi x \alpha \pi^{\prime}\right)$. Reduction on proof-terms is defined by the following rules that eliminate cuts step by step.

$$
\begin{gathered}
\left(\lambda \alpha \pi_{1} \pi_{2}\right) \triangleright\left[\pi_{2} / \alpha\right] \pi_{1} \\
f s t\left(\left\langle\pi_{1}, \pi_{2}\right\rangle\right) \triangleright \pi_{1} \\
\operatorname{snd}\left(\left\langle\pi_{1}, \pi_{2}\right\rangle\right) \triangleright \pi_{2} \\
\left(\delta i\left(\pi_{1}\right) \alpha \pi_{2} \beta \pi_{3}\right) \triangleright\left[\pi_{1} / \alpha\right] \pi_{2}
\end{gathered}
$$

INRIA 


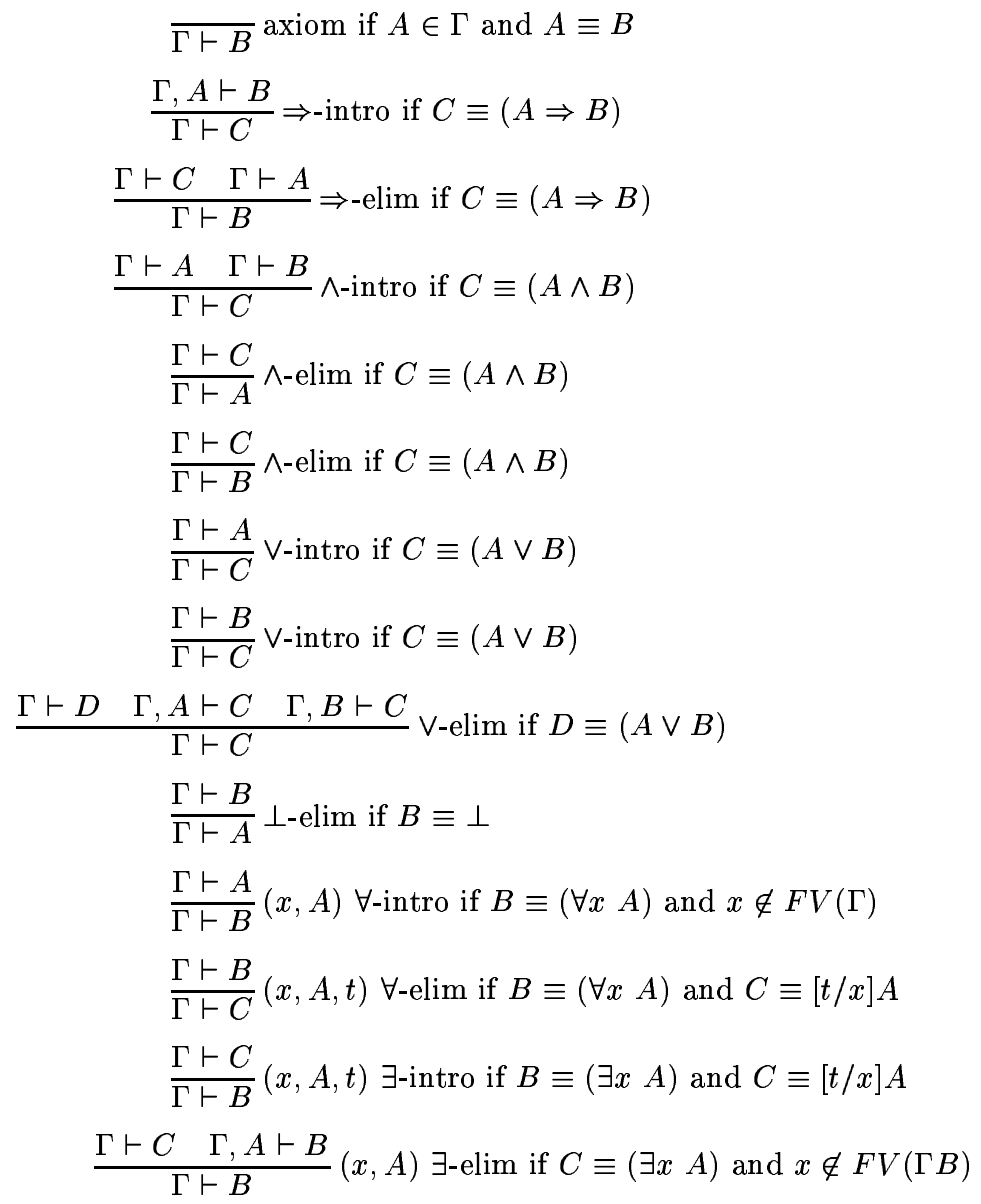

Figure 1: Natural deduction modulo 


$$
\begin{gathered}
\left(\delta j\left(\pi_{1}\right) \alpha \pi_{2} \beta \pi_{3}\right) \triangleright\left[\pi_{1} / \beta\right] \pi_{3} \\
(\lambda x \pi t) \triangleright[t / x] \pi \\
\left(\text { exelim }\left\langle t, \pi_{1}\right\rangle \alpha x \pi_{2}\right) \triangleright\left[t / x, \pi_{1} / \alpha\right] \pi_{2}
\end{gathered}
$$

Proofs in deduction modulo, are written as in first-order natural deduction, and the proof reduction rules are the same.

Proofs built with the folding rule are written $\pi \uparrow$ and proofs built with the unfolding rule are written $\pi \downarrow$. The corresponding reduction rule is

$$
\pi \uparrow \downarrow \triangleright \pi
$$

\section{Translations}

As already said, a sequent $\Gamma \vdash A$ is provable in deduction with the folding and unfolding rules if and only if the sequent $\mathcal{T}, \Gamma \vdash A$ is provable in first-order natural deduction. The sequent $\Gamma \vdash A$ is also provable in deduction modulo if and only if the sequent $\mathcal{T}, \Gamma \vdash A$ is provable in first-order natural deduction. Hence the sequent $\Gamma \vdash A$ is provable in deduction with the folding and unfolding rules if and only if it is provable in deduction modulo.

This can also be proved directly. If $\pi$ is a proof of $\Gamma \vdash A$ in deduction with the folding and unfolding rules, then the proof $\pi^{-}$obtained by erasing the folding and unfolding steps in $\pi$ yields a proof in deduction modulo. The converse is a little bit more difficult. Indeed, if the proposition $B$ unfolds to $B^{\prime}$ then the proposition $A \wedge B$ and $A \wedge B^{\prime}$ are congruent and hence the sequent $A \wedge B \vdash A \wedge B^{\prime}$ has a trivial proof in deduction modulo, using only the axiom rule. But the unfolding rule does not apply to the proposition $A \wedge B$, but only to the proposition $B$. Hence the proof of $A \wedge B^{\prime}$ must be written $\langle f s t(\alpha), \operatorname{snd}(\alpha) \downarrow\rangle$ where the variable $\alpha$ is $\eta$-expanded so that the unfolding rule can apply to the proposition $B$. This justifies the need of the following lemma.

Lemma 4.1 If the proposition $A$ converts to $B$ in one step (we write this $A \leftrightarrow^{1} B$ ), then there are proofs of $A \Rightarrow B$ and $B \Rightarrow A$ in deduction with the folding and unfolding rules. These proofs are called conversion steps.

Proof. By induction over the structure of $A$.

- If $A$ is atomic, then $A$ unfolds to $B$ and we take the proofs $\lambda \alpha \alpha \downarrow$ and $\lambda \alpha \alpha \uparrow$.

- If $A=A_{1} \Rightarrow A_{2}$ then $B=B_{1} \Rightarrow B_{2}$. We have either $A_{1}=B_{1}$ and $A_{2} \leftrightarrow^{1} B_{2}$ or $A_{1} \leftrightarrow^{1} B_{1}$ and $A_{2}=B_{2}$. By induction hypothesis we have in both cases proofs $\rho_{1}$ and $\rho_{1}^{\prime}$ of $A_{1} \Rightarrow B_{1}$ and $B_{1} \Rightarrow A_{1}$ and $\rho_{2}$ and $\rho_{2}^{\prime}$ of $A_{2} \Rightarrow B_{2}$ and $B_{2} \Rightarrow A_{2}$. We take $\lambda \alpha \lambda \beta\left(\rho_{2}\left(\alpha\left(\rho_{1}^{\prime} \beta\right)\right)\right)$ and $\lambda \alpha \lambda \beta\left(\rho_{2}^{\prime}\left(\alpha\left(\rho_{1} \beta\right)\right)\right)$.

- If $A=A_{1} \wedge A_{2}$ then $B=B_{1} \wedge B_{2}$. We have either $A_{1}=B_{1}$ and $A_{2} \leftrightarrow^{1} B_{2}$ or $A_{1} \leftrightarrow^{1} B_{1}$ and $A_{2}=B_{2}$. By induction hypothesis we have in both cases proofs $\rho_{1}$ and $\rho_{1}^{\prime}$ of $A_{1} \Rightarrow B_{1}$ and $B_{1} \Rightarrow A_{1}$ and $\rho_{2}$ and $\rho_{2}^{\prime}$ of $A_{2} \Rightarrow B_{2}$ and $B_{2} \Rightarrow A_{2}$. We take $\lambda \alpha\left\langle\left(\rho_{1} f s t(\alpha)\right),\left(\rho_{2} \operatorname{snd}(\alpha)\right)\right\rangle$ and $\lambda \alpha\left\langle\left(\rho_{1}^{\prime} f s t(\alpha)\right),\left(\rho_{2}^{\prime} \operatorname{snd}(\alpha)\right)\right\rangle$. 
- If $A=A_{1} \vee A_{2}$ then $B=B_{1} \vee B_{2}$. We have either $A_{1}=B_{1}$ and $A_{2} \leftrightarrow^{1} B_{2}$ or $A_{1} \leftrightarrow^{1} B_{1}$ and $A_{2}=B_{2}$. By induction hypothesis we have in both cases proofs $\rho_{1}$ and $\rho_{1}^{\prime}$ of $A_{1} \Rightarrow B_{1}$ and $B_{1} \Rightarrow A_{1}$ and $\rho_{2}$ and $\rho_{2}^{\prime}$ of $A_{2} \Rightarrow B_{2}$ and $B_{2} \Rightarrow A_{2}$. We take $\lambda \alpha\left(\delta \alpha \beta i\left(\rho_{1} \beta\right) \gamma j\left(\rho_{2} \gamma\right)\right)$ and $\lambda \alpha\left(\delta \alpha \beta i\left(\rho_{1}^{\prime} \beta\right) \gamma j\left(\rho_{2}^{\prime} \gamma\right)\right)$.

- Since $A$ is reducible, it cannot be $\perp$

- If $A=\forall x A_{1}$ then $B=\forall x B_{1}$ and we have $A_{1} \leftrightarrow^{1} B_{1}$. By induction hypothesis we have proofs $\rho_{1}$ and $\rho_{1}^{\prime}$ of $A_{1} \Rightarrow B_{1}$ and $B_{1} \Rightarrow A_{1}$. We take $\lambda \alpha \lambda x\left(\rho_{1}(\alpha x)\right)$ and $\lambda \alpha \lambda x\left(\rho_{1}^{\prime}(\alpha x)\right)$.

- If $A=\exists x A_{1}$ then $B=\exists x B_{1}$ and we have $A_{1} \leftrightarrow^{1} B_{1}$. By induction hypothesis we have proofs $\rho_{1}$ and $\rho_{1}^{\prime}$ of $A_{1} \Rightarrow B_{1}$ and $B_{1} \Rightarrow A_{1}$. We take $\lambda \alpha$ (exelim $\alpha x \beta\left\langle x,\left(\rho_{1} \beta\right)\right\rangle$ ) and $\lambda \alpha$ (exelim $\left.\alpha x \beta\left\langle x,\left(\rho_{1}^{\prime} \beta\right)\right\rangle\right)$.

Corollary 4.1 If $A \equiv A^{\prime}$ and $\pi$ is a proof of $\Gamma \vdash A$, then there is a proof of $\Gamma \vdash A^{\prime}$ of the form $\left(\rho_{n} \ldots\left(\rho_{1} \pi\right) \ldots\right)$ where $\rho_{1}, \ldots, \rho_{n}$ are conversion steps. Such a proof is called a transformation of $\pi$.

Proposition 4.1 The propositions provable in deduction with the folding and unfolding rules and the propositions provable in deduction modulo are the same.

Proof. Erasing the folding and unfolding steps transforms any proof $\pi$ in deduction with the folding and unfolding rules into a proof $\pi^{-}$in deduction modulo. Converselly, if a sequent has a proof $\pi$ in deduction modulo, then we can build a proof $\pi^{+}$in deduction with the folding and unfolding rules inserting sequences of conversion steps when needed.

\section{Folding-unfolding cuts and cuts modulo}

We now prove that cut elimination terminates in deduction with the folding and unfolding rules if and only if cut elimination terminates in deduction modulo.

Lemma 5.1 Let $A$ and $A^{\prime}$ be two propositions such that $A \equiv A^{\prime}$ and $A$ and $A^{\prime}$ are either two implications, two conjunctions, two disjunctions, two contradictions, two universal quantifications or two existential quantifications. Let $\pi$ be a proof of $\Gamma \vdash A$ and $\pi^{\prime}$ a proof of $\Gamma \vdash A^{\prime}$ that is a transformation of $\pi$.

If $\pi$ is an introduction, then $\pi^{\prime}$ reduces to an introduction $\pi^{\prime \prime}$ and the subproofs of $\pi^{\prime \prime}$ are transformations of subproofs of $\pi$.

Proof. By induction on the length of the transformation from $\pi$ to $\pi^{\prime}$. The result is obvious if $\pi^{\prime}=\pi$. Otherwise, the proof $\pi^{\prime}$ has the form $\left(\rho_{n+1}\left(\rho_{n} \ldots\left(\rho_{1} \pi\right) \ldots\right)\right)$. The proof $\left(\rho_{n} \ldots\left(\rho_{1} \pi\right) \ldots\right)$ is a proof of a proposition $A^{\prime \prime}$ that is either atomic or has the same head symbol as $A$. 
If $A^{\prime \prime}$ is atomic then $n \neq 0$ and $\left(\rho_{n-1} \ldots\left(\rho_{1} \pi\right) \ldots\right)$ is a proof of $A^{\prime}, \rho_{n}=\lambda \alpha \alpha \uparrow$ and $\rho_{n+1}=\lambda \alpha \alpha \downarrow$. By induction hypothesis, $\left(\rho_{n-1} \ldots\left(\rho_{1} \pi\right) \ldots\right)$ reduces to an introduction $\pi^{\prime \prime}$ and the subproofs of $\pi^{\prime \prime}$ are transformations of the subproofs of $\pi$. The proof $\pi^{\prime}$ reduces to $\pi^{\prime \prime} \uparrow \downarrow$ and then to $\pi^{\prime \prime}$.

Otherwise $A^{\prime \prime}$ has the same head symbol as $A$. By induction hypothesis, the proof $\left(\rho_{n} \ldots\left(\rho_{1} \pi\right) \ldots\right)$ reduces to $\lambda \alpha \pi_{1}^{\prime \prime}$ (resp. $\left.\left\langle\pi_{1}^{\prime \prime}, \pi_{2}^{\prime \prime}\right\rangle, i\left(\pi_{1}^{\prime \prime}\right), j\left(\pi_{1}^{\prime \prime}\right), \lambda x \pi_{1}^{\prime \prime},\left\langle t, \pi_{1}^{\prime \prime}\right\rangle\right)$, the subproofs are transformations of the subproofs of $\pi$ and $\rho_{n+1}$ has the form

$$
\lambda \alpha \lambda \beta\left(\tau_{2}\left(\alpha\left(\tau_{1}^{\prime} \beta\right)\right)\right)
$$

(resp.

$$
\begin{gathered}
\lambda \alpha\left\langle\left(\tau_{1} f s t(\alpha)\right),\left(\tau_{2} \text { snd }(\alpha)\right)\right\rangle \\
\lambda \alpha\left(\delta \alpha \beta i\left(\tau_{1} \beta\right) \gamma j\left(\tau_{2} \gamma\right)\right) \\
\lambda \alpha\left(\delta \alpha \beta i\left(\tau_{1} \beta\right) \gamma j\left(\tau_{2} \gamma\right)\right) \\
\lambda \alpha \lambda x\left(\tau_{1}(\alpha x)\right) \\
\lambda \alpha\left(\text { exelim } \alpha x \beta\left\langle x,\left(\tau_{1} \beta\right)\right\rangle\right)
\end{gathered}
$$

). Thus, the proof $\pi^{\prime}$ reduces to an introduction $\pi^{\prime \prime}$ and the subproofs of $\pi^{\prime \prime}$ are transformations of subproofs of $\pi$.

Proposition 5.1 Cut elimination terminates in deduction with folding and unfolding rule if and only of cut elimination modulo terminates.

Proof. Assume that cut elimination modulo terminates and consider a cut elimination sequence $\pi_{1}, \pi_{2}, \ldots$ in deduction with the folding and unfolding rules. Each $\pi_{i+1}$ is obtained from $\pi_{i}$ either by reducing a logical cut or a folding-unfolding cut and only a finite number folding-unfolding cut reductions can be performed consecutively (as the size of proofs reduces when we reduce such a cut). When $\pi_{i+1}$ is obtained by reducing a logical cut in $\pi_{i}$ then $\pi_{i+1}^{-}$is obtained by reducing a cut in $\pi_{i}^{-}$and when $\pi_{i+1}$ is obtained by reducing a foldingunfolding cut in $\pi_{i}$ then $\pi_{i+1}^{-}=\pi_{i}^{-}$. Hence in the sequence $\pi_{1}^{-}, \pi_{2}^{-}, \ldots$ in deduction modulo each proof is either obtained by reducing a cut in the previous, or is equal to the previous and only a finite number of consecutive proofs can be equal. As cut elimination terminates in deduction modulo, this sequence is finite and so is the sequence $\pi_{1}, \pi_{2}, \ldots$.

Converselly, assume that cut elimination terminates in deduction with the folding and unfolding rules and consider a proof-reduction sequence $\pi_{1}, \pi_{2}, \ldots$ in deduction modulo. The proof $\pi_{1}$ contains a redex. In $\pi_{1}^{+}$a conversion steps may have been inserted between the introduction rule and the elimination rule of this redex. But, by lemma 5.1, reducing the conversion steps applied to the introduction permutes the introduction and the conversion steps. Thus, $\pi_{1}^{+}$reduces to $\pi_{2}^{+}$in at least one step. Hence the sequence $\pi_{1}^{+}, \pi_{2}^{+}, \ldots$ is finite and so is the sequence $\pi_{1}, \pi_{2}, \ldots$ 


\section{Comparing deduction modulo and deduction with the folding and unfolding rules}

In [7] we have shown that a theory modulo had the cut elimination property if it had some kind of many-valued model (whose truth values are sets of proofs) called a pre-model and we have shown that large classes of theories modulo had the cut elimination property. As cut elimination is equivalent in deduction with the folding and unfolding rules and in deduction modulo, these tools can be adapted to prove cut elimination in deduction with the folding and unfolding rules.

Alternatively, theories usually presented in deduction with the folding and unfolding rules can equivalently be presented in deduction modulo.

Proofs in deduction modulo are more compact than proofs in deduction with the folding and unfolding rules. First, because the folding and unfolding steps are left implicit in deduction modulo, but also because in deduction with the folding and unfolding rules, as show above, proofs need to be $\eta$-expanded so that the folding and unfolding rules may be applied. This $\eta$-expansion could be avoided if we extended the folding and unfolding rules to a conversion rule

$$
\frac{\Gamma \vdash A}{\Gamma \vdash B} \text { conversion if } A \equiv B
$$

with arbitrary propositions $A$ and $B$. But, then lemma 5.1 would not hold anymore and a conversion step inserted between a introduction and an elimination could block a cut, as in the proof (with $B \equiv B^{\prime}$ )

$$
\frac{\frac{A, B \vdash A \quad A, B \vdash B}{A, B \vdash A \wedge B} \wedge \text {-intro }}{\frac{A, B \vdash A \wedge B^{\prime}}{A, B \vdash A} \wedge \text {-elim }}
$$

We would then need to extend the notion of cut and define a cut as a sequence formed with an introduction rule, a sequence of conversion rules and an elimination rule [4], i.e. essentially as a cut modulo.

At last, deduction modulo is more general than deduction with the folding and unfolding rules, as it does not require that an atomic proposition unfolds to at most one proposition. This permits in particular to include, besides equivalences between propositions such as $x \times y=0 \equiv x=0 \vee y=0$, equivalence between terms such as $x+0 \equiv x$. Then a proposition such as $(x+0) \times(y+0)=0$ may be equivalent to many propositions such as $x \times(y+0)=0$, $(x+0) \times y=0, x+0=0 \vee y+0=0, x=0 \vee y=0, \ldots$ while this is not possible in deduction with the folding and unfolding rules.

\section{References}

[1] S.C. Bailin. A normalization theorem for set theory. The Journal of Symbolic Logic, 53, 3 (1988) pp. 673-695 
[2] M. Crabbé. Non-normalisation de la théorie de Zermelo, manuscript (1974).

[3] M. Crabbé. Stratification and cut-elimination. The Journal of Symbolic Logic, 56 (1991) pp. 213-226.

[4] G. Dowek, Proof normalization for a first-order formulation of higher-order logic, E.L. Gunter and A. Felty (Eds.), Theorem Proving in Higher-order Logics, Lecture notes in computer science 1275, Springer-Verlag (1997), pp. 105-119. Rapport de Recherche 3383, INRIA (1998).

[5] G. Dowek, Th. Hardin, and C. Kirchner. Theorem proving modulo. Rapport de Recherche 3400, Institut National de Recherche en Informatique et en Automatique (1998). To appear in Journal of Automated Reasoning.

[6] G. Dowek, Th. Hardin, and C. Kirchner. HOL- $\lambda \sigma$ an intentional first-order expression of higher-order logic. In P. Narendran and M. Rusinowitch, editors, Rewriting Techniques and Applications, number 1631 in Lecture Notes in Computer Science, Springer-Verlag, (1999) pp. 317-331.

[7] G. Dowek and B. Werner. Proof normalization modulo. In Types for proofs and programs 98, volume 1657 of Lecture Notes in Computer Science, Springer-Verlag, (1999), pp. 62-77.

[8] J. Ekman, Normal proofs in set theory, Doctoral thesis, Chalmers University of Technology and University of Göteborg (1994).

[9] L. Hallnäs, On normalization of proofs in set theory, Doctoral thesis, University of Stockholm (1983).

[10] D. Prawitz. Natural deduction, a proof-theoretical study. Almqvist \& Wiksell (1965). 


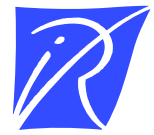

Unité de recherche INRIA Rocquencourt Domaine de Voluceau - Rocquencourt - BP 105 - 78153 Le Chesnay Cedex (France)

Unité de recherche INRIA Lorraine : LORIA, Technopôle de Nancy-Brabois - Campus scientifique 615, rue du Jardin Botanique - BP 101 - 54602 Villers-lès-Nancy Cedex (France)

Unité de recherche INRIA Rennes : IRISA, Campus universitaire de Beaulieu - 35042 Rennes Cedex (France)

Unité de recherche INRIA Rhône-Alpes : 655, avenue de l'Europe - 38330 Montbonnot-St-Martin (France)

Unité de recherche INRIA Sophia Antipolis : 2004, route des Lucioles - BP 93 - 06902 Sophia Antipolis Cedex (France) 